\title{
Anabases
}

ANABASES Traditions et réceptions de l'Antiquité

5 | 2007

Varia

\section{Johann Gustav DROYSEN, Histoire de l'Hellénisme}

\section{Luciano Canfora}

\section{OpenEdition \\ Journals}

\section{Edizione digitale}

URL: http://journals.openedition.org/anabases/3257

DOI: 10.4000/anabases.3257

ISSN: 2256-9421

\section{Editore}

E.R.A.S.M.E.

\section{Edizione cartacea}

Data di pubblicazione: 1 marzo 2007

Paginazione: 277-280

ISSN: 1774-4296

Notizia bibliografica digitale

Luciano Canfora, « Johann Gustav droysen, Histoire de l'Hellénisme », Anabases [Online], 5 | 2007, Messo online il 01 décembre 2011, consultato il 20 septembre 2020. URL : http://journals.openedition.org/ anabases/3257 ; DOI : https://doi.org/10.4000/anabases.3257

Questo documento è stato generato automaticamente il 20 settembre 2020.

(c) Anabases 


\title{
Johann Gustav DROYSEN, Histoire de l'Hellénisme
}

\author{
Luciano Canfora
}

\section{NOTIZIA}

Johann Gustav DROYSEN, Histoire de l'Hellénisme (édition intégrale),Traduit de l'allemand sous la dir. d'Auguste Bouché-Leclercq, Préface, note sur la traduction, bibliographie (p. 5-82) par Pascal PAYEN, Grenoble, Jérôme Millon, 2 vol., 2005, 1769 p.

75 euros / ISBN 2-84137-172-7

1 Pascal Payen ha il merito di riproporre in accessibile e molto ben curata edizione moderna la bella traduzione della Storia dell'Ellenismo di Johann Gustav Droysen curata a suo tempo, e in feconda dialettica con lo «stile » del suo autore, da Auguste BouchéLeclercq. Tappa dell'incontro-scontro franco-tedesco nel corso di tutto il xIx secolo, questa traduzione ha giovato moltissimo alla diffusione fuori della Germania dell'opera più importante di Droysen. Inoltre Payen ricostruisce in una densa e ben documentata introduzione le tappe del lavoro di Droysen, le sue ambizioni ed il suo parziale fallimento; nonché il significato che l'opera ebbe nello svecchiamento in senso anticlassicistico degli studi sul mondo greco ed ellenizzato.

2 La questione aperta, nella comprensione di quest'opera, non è più tanto quella della sua portata innovativa (al di là dell'influsso che su Droysen avevano avuto le ricerche precorritrici di Niebuhr) quanto la mutazione che essa subì con la seconda edizione (1877/1878). La seconda edizione è quella che abitualmente si adopera e che è stata tradotta da Bouché-Leclercq. E' dunque di tale edizione che ci si serve tuttora nei limiti in cui l'opera continua ad essere adoperata per il suo contenuto storico-ricostruttivo. Nondimeno, soprattutto per merito di Arnaldo Momigliano, e per l'esattezza del giovanissimo Momigliano della prima metà degli anni Trenta, resta ineludibile la constatazione che l'opera fu profondamente trasformata da Droysen nel passaggio dalla prima alla seconda edizione. Momigliano mise in luce la maggiore originalità del pur 
acerbo «primo » Alessandro e la larghezza di orizzonte storiografico della cosiddetta "prefazione privata " all'Ellenismo rispetto alla ben minore audacia intellettuale della seconda edizione. E' vero che il giovanissimo Momigliano risentiva di una forte impronta filosofica ; ma questo non era affatto un danno, anzi aiutava a cogliere aspetti che di solito non si guardano in profondità per una male intesa ritrosìa a «sporcarsi le mani » con l'interferenza della filosofia soprattutto idealistica, nella storiografia tedesca dell'Ottocento. Che ci fosse anche una adesione intellettuale di Momigliano nei confronti del "clima spirituale» in cui aveva operato, e creato, il giovane Droysen è altro discorso. Sembra dunque resistere l'intuizione di Momigliano anche di fronte alle critiche di Benedetto Bravo, il quale, nel suo ben noto saggio su Droysen, ha voluto accorciare le distanze tra il primo e il secondo Ellenismo. Su questo punto sono ben fondate e del tutto condivisibili le considerazioni di Payen (p.52 e nota 233) sul sostanziale mantenimento, da parte di Momigliano, anche negli scritti degli anni 1970/1980, delle sue originarie posizioni.

3 Così come ha ben ragione Payen nel rilevare - sulla scia di Momigliano - il maggiore «prussianesimo» del secondo Ellenismo: accrescimento della prospettiva politica «attuale» che trova riscontro, ovviamente, nell'opus maximum - e monumentale ma anch'esso incompiuto - dell'ultimo Droysen sulla politica estera prussiana.

Quello della Macedonia-Prussia è stato ed è rimasto un filo conduttore della storia intellettuale e pratica tedesca ben oltre Droysen. Un filo conduttore di cui giova qui ricordare alcune ulteriori tappe.

5 «Nicht Demosthenes mit seinen ephemeren Reden und seinen papierenen Demonstrationen gegen Alexander den Großen, sondern Alexander der Große, der Begründer jener Kultur, aus der das Christentum und die Organisation des Augusteischen Staates entstanden ist, muß gekannt werden ». Questo non molto conosciuto pensiero di Ulrich von Wilamowitz-Moellendorff si legge nel suo intervento alla Schulkonferenz berlinese (6-8 giugno 1900) voluta da Guglielmo II per l'avvio di una radicale riforma scolastica (Verhandlungen über Fragen des höheren Unterrichts, Halle 1901, p. 90). Nonostante sia parso a suo tempo «iconoclastico» («non possiamo rinunciare a Demostene !» replicarono i professori di ginnasio), questo intervento corrisponde ad un cliché : quello della esaltazione dell'Ellenismo e del suo vero fondatore, Alessandro. $\mathrm{Ci}$ sono infatti elementi che stridono nelle parole che abbiamo ora ricordato. Per esempio : perché Wilamowitz, intellettualmente lontano dal cristianesimo qui esalta Alessandro perché avrebbe "preparato" il cristianesimo? Evidentemente è un omaggio a Droysen. E ancora : come può affermare che «l'organizzazione » dell'impero di Alessandro avesse costituito un modello per quello di Augusto? Wilamowitz fa propria una radicale esaltazione di Alessandro come fattore sia spirituale che politico destinato ad un grande futuro - creatore dell'Ellenismo -, e svaluta Demostene («discorsi effimeri e dimostrazioni cartacee»!), come simbolo di tutto quanto l'Ellenismo spazzò via : innanzi tutto la vecchia mentalità gretta dell'angusto orizzonte « cittadino ».

Effettivamente, tra i primi a fare le spese della «scoperta » dell'Ellenismo fu appunto Demostene. Non fu però un processo del tutto lineare. Per esempio, pochi decenni prima di Droysen, l'oratoria demostenica era stata usata come alimento (oratorio) della rinascita, in senso antifrancese, della "nazione tedesca» (Fichte, Jacobs). In quel momento, e in tale prospettiva, Napoleone corrispondeva a Filippo il Macedone mentre la Prussia in lotta contro di lui nonché epicentro di una rinascita nazionale di tutta (o 
quasi) la Germania corrispondeva all'Atene di Demostene. Che un secolo più tardi (1914/1915) Wilamowitz esaltasse proprio $\mathrm{i}$ «Freiheitskriege » del tempo di Fichte e di Jacobs per chiamare i tedeschi alla lotta contro l'Intesa è solo uno degli innumerevoli aspetti dell'inesauribile «ironia della storia». E per giunta fu proprio una nuova generazione di storici prussiani (K.J. Beloch soprattutto) che liquidò il libro di Droysen come « romanticheria».

7 La contrapposizione Demostene/sovrani macedoni aveva una matrice assai remota. Era già presente nell'opera storiografica di Teopompo di Chio, il grande storico di Filippo, il quale aveva attribuito a Filippo il rango ed il ruolo di « uomo più grande che l'Europa abbia prodotto ", laddove aveva inquadrato Demostene in luce molto negativa in quel decimo libro delle Storie Filippiche che ebbe anche circolazione autonoma col titolo Sui demagoghi ateniesi.

Vitalità di un mito eminentemente ideologico: la polarità Demostene/sovrani macedoni è ancora viva in epoca nazista. Basti considerare le reazioni al Demosthenes di Werner Jaeger (1938). Non va dimenticato che il titolo esatto dell'opera è quello inglese (The Origin and Growth of His Policy): esso spiega perché il libro proceda approfonditamente fino a Cheronea (338 a.C.), e solo in iscorcio consideri l'ultima fase, cioè i quindici anni fino alla morte di Alessandro e dello stesso Demostene.

9 Appena diffuso, in California (1938) e a Berlino (1939), il Demosthenes fu oggetto di due importanti recensioni, rispettivamente all'edizione americana e a quella tedesca : di Kurt von Fritz («American Historical Review» 44, 1939) e di Helmut Berve («Göttingische Gelehrte Anzeigen » 202, novembre 1940). Essenziale e politicamente consenziente col pensiero di Jaeger la prima ; durissima, a tratti sarcastica, ma molto analitica, la seconda.

10 La tesi centrale di Jaeger è contro-corrente - scriveva von Fritz - : egli è persuaso infatti della sostanziale giustezza della politica demostenica («gli Ateniesi non seguirono i suoi suggerimenti quando il successo sarebbe stato sicuro »). Ma tale rivalutazione della concretezza politica di Demostene, di solito presentato come un sognatore $o$ al più come un venduto alla Persia, si discostava molto dalla diagnosi vincente (Droysen, Beloch). «Beloch - scrive von Fritz -, an outstanding representative of the positivistic conception of history, in the introduction to his Griechische Geschichte vehemently impugns the view that it is great men who make history. Historical changes, in his opinion, is brought about through the subconscious tendencies of the anonymous masses. A man, therefore, who opposed the general tendency of his age (which, in the case of Demosthenes, led from the Hellenic city-State to Hellenistic monarchy) appeared to him somewhat lacking in political insight ». E aggiungeva : nell'odierna Germania gli storici pensano daccapo che sia il grande uomo ("the hero, the leader ») che fa la storia, " and the judgement on the opponents of the man of destiny - in Demosthenes' case Philip of Macedon - has become still harsher» (p. 583). Eppure ironizzava - l'eroe, se non trovasse oppositori non potrebbe « display his heroism »!

11 Il lunghissimo intervento di Berve è un vero e proprio atto d'accusa. Egli deprime il libro definendolo " una serie di conferenze », e deride la pretesa di Jaeger di porsi nel solco degli interpreti di Demostene che furono anche "uomini d'azione». L'attacco mira innanzi tutto a demolire l'immagine « troppo positiva » dell'Atene del IV secolo: ammettere la presenza di "ethische Kräfte » nell'Atene del IV secolo significa - per Berve - collocare «Streben und Leistungen » di Demostene in una prospettiva erronea. Addirittura Jaeger viene accusato di accettare l'erronea visione demostenica dei 
Macedoni come non Greci (p. 466-467). Naturalmente è Filippo al centro della dimostrazione, e Berve assicura che l'origine greca della «stirpe » di Filippo era inconfutabilmente ancorata «in seinem Griechentum». Jaeger è invischiato («befangen ») nel « Kreis der demosthenischen Gedanken», nonostante la «dura critica » cui Droysen e Beloch avevano sottoposto l'opera di quel politico (p. 468). I nomi di Droysen e Beloch vengono fatti più volte e il principale rimprovero rivolto a Jaeger è per l'appunto di essersi distaccato dalla ormai consolidata "Behandlung " della politica demostenica, sviluppata dalla "deutsche Geschichtswissenschaft » (p. 471). Non meno duro è Fritz Taeger su «Gnomon» del 1941, la cui recensione si chiude un po' ruvidamente con la domanda - posta a suo tempo da Droysen - se davvero Demostene, persino nella sempre esaltata Terza Filippica, possa essere definito "patriota", e non piuttosto fiancheggiatore della politica persiana. Non è superfluo ricordare che nello stesso anno del Demosthenes di Jaeger era uscito a Monaco il Filippo di F.R. Wüst, in linea con la valutazione « prussiana » del sovrano.

La discussione su Demostene e Filippo, assunti quasi a metafora di conflitti attuali, si era sviluppata anche in Italia. Il Demostene di Piero Treves (1933) ed il Filippo il Macedone (1938) di Arnaldo Momigliano rendono bene questa polarità. Proprio dall'ambiente del fascismo culturale italiano venne il più duro attacco contro Jaeger. È la lunga e aspra recensione scritta da Gennaro Perrotta sulla rivista del ministro dell'Educazione Nazionale Giuseppe Bottai «Primato »(a. III, 22, 15 novembre 1942). Lì viene messo sotto accusa il « classicismo ", che ha consacrato a Demostene « un culto eroico ", viene definito il libro di Jaeger «prova della funesta immortalità del classicismo ", sbeffeggiato Piero Treves come autore di « uno sconclusionato libretto su Demostene e la libertà dei Greci », vilipeso il concetto di libertà come autonomia, esaltata la « necessità e razionalità della storia » che sta alla base del trionfo di Filippo contro la «libertà grettamente municipale di Atene ». Il tutto nel nome di Droysen, di Beloch e della vera politica « che non sa che farsi della retorica ». Il tono è sovreccitato e trasparentemente politico: Treves, come ebreo, aveva dovuto riparare in Inghilterra per le leggi razziali del 1938, e la guerra hitleriana stava facendo strame della « libertà come autonomia ». Ecco un chiaro segno perdurante della battaglia intorno all'iniziatore dell'Ellenismo.

\section{AUTORI}

\section{LUCIANO CANFORA}

Università di Bari

l.canfora@lettere.unibari.it 